Research Article

\title{
New Iteration Scheme for Approximating a Common Fixed Point of a Finite Family of Mappings
}

\author{
F. O. Isiogugu $\mathbb{D}^{1,2}$ C. Izuchukwu, ${ }^{1}$ and C. C. Okeke ${ }^{1}$ \\ ${ }^{1}$ School of Mathematics, Statistics and Computer Science, University of KwaZulu-Natal, Durban, South Africa \\ ${ }^{2}$ Department of Mathematics, University of Nigeria, Nsukka, Nigeria
}

Correspondence should be addressed to F. O. Isiogugu; felicia.isiogugu@unn.edu.ng

Received 28 July 2019; Accepted 2 April 2020; Published 1 May 2020

Guest Editor: Aftab Hussain

Copyright (c) 2020 F. O. Isiogugu et al. This is an open access article distributed under the Creative Commons Attribution License, which permits unrestricted use, distribution, and reproduction in any medium, provided the original work is properly cited.

We introduce a new algorithm (horizontal algorithm) in a real Hilbert space, for approximating a common fixed point of a finite family of mappings, without imposing on the finite family of the control sequences $\left\{\left\{\varsigma_{n}^{i}\right\}_{n=1}^{\infty}\right\}_{i=1}^{N}$, the condition that $\sum_{i=1}^{N} \varsigma_{n}^{i}=1$, for each $n \geq 1$. Furthermore, under appropriate conditions, the horizontal algorithm converges both weakly and strongly to a common fixed point of a finite family of type-one demicontractive mappings. It is also applied to obtain some new algorithms for approximating a common solution of an equilibrium problem and the fixed point problem for a finite family of mappings. Our work is a contribution to ongoing research on iteration schemes for approximating a common solution of fixed point problems of a finite family of mappings and equilibrium problems.

\section{Introduction}

Let $Y$ be a nonempty set and $S: Y \longrightarrow Y$ be a mapping. A point $y \in Y$ is called a fixed point of $S$ if $y=S y$. If $S: Y \longrightarrow 2^{Y}$ is a multivalued mapping, then $y$ is a fixed point of $S$ if $y \in S y . y$ is called a strict fixed point of $S$ if $S y=\{y\}$. The set $F(S)=\{y \in D(S): y \in S y\} \quad$ (respectively, $F(S)=\{y \in D(S): y=S y\})$ is called the set of fixed points of the multivalued (respectively, single-valued) mapping $S$, while the set $F_{s}(S)=\{y \in D(S): S y=\{y\}\}$ is called the set of strict fixed points of $S$.

Let $Y$ be a normed space. A subset $K$ of $Y$ is called proximinal if for each $y \in Y$, there exists $k \in K$ such that

$$
\|y-k\|=\inf \{\|y-w\|: w \in K\}=d(y, K) .
$$

It is known that every convex closed subset of a uniformly convex Banach space is proximinal. We shall denote the family of all nonempty closed and bounded subsets of $Y$ by $C B(Y)$, the family of all nonempty subsets of $Y$ by $2^{Y}$, and the family of all proximinal subsets of $Y$ by $P(Y)$, for a nonempty set $Y$.

Let $\mathscr{D}$ denote the Hausdorff metric induced by the metric $d$ on $Y$, that is, for every $A, B \in C B(Y)$,

$$
\mathscr{D}(A, B)=\max \left\{\sup _{a \in A} d(a, B), \sup _{b \in B} d(b, A)\right\} .
$$

Let $Y$ be a normed space and $S: D(S) \subseteq Y \longrightarrow 2^{Y}$ be a multivalued mapping on $Y . S$ is called $L-$ Lipschitzian if there exists $L \geq 0$ such that, for all $x, y \in D(S)$,

$$
\mathscr{D}(S x, S y) \leq L\|x-y\| .
$$

In (3), if $L \in[0,1)$, then $S$ is a contraction, while $S$ is nonexpansive if $L=1 . S$ is called quasi-nonexpansive if $F(S) \neq \varnothing$ and for all $p \in F(S)$,

$$
\mathscr{D}(S x, S p) \leq\|x-p\| \text {. }
$$

Clearly, every nonexpansive mapping with the nonempty fixed point set is quasi-nonexpansive. The multivalued mapping $S$ is $k$-strictly pseudo-contractive-type of Isiogugu [1] using the terminology of Browder and Petryshen [2] for single-valued pseudo-contractive mapping and Markin [3] for the monotone operator if there exists $k \in[0,1)$ such that given any pair $x, y \in D(S)$ and $u \in S x$, there exists $v \in S y$ satisfying $\|u-v\| \leq \mathscr{D}(S x, S y)$ and

$$
\mathscr{D}^{2}(S x, S y) \leq\|x-y\|^{2}+k\|x-u-(y-v)\|^{2} .
$$


If $k=1$ in (5), then $S$ is pseudo-contractive-type, while $S$ is nonexpansive-type if $k=0$. Every multivalued nonexpansive mapping $S: D(S) \subseteq Y \longrightarrow P(Y)$ is nonexpansivetype. $S$ is of type-one in the sense of Isiogugu et al. [4] if given any pair $x, y \in D(S)$, then

$$
\|u-v\| \leq \mathscr{D}(S x, S y), \quad \text { for all } u \in P_{S} x, v \in P_{S} y,
$$

where $P_{S} x:=\{u \in S x:\|u-x\|=d(x, S x)\} . S$ is called a multivalued demicontractive in the sense of Isiogugu and Osilike [5] using the terminology of Hicks and Kubicek [6] for single-valued demicontractive if $F(S) \neq \varnothing$ and for all $p \in F(S)$ and $x \in D(S)$, there exists $k \in[0,1)$ such that

$$
\mathscr{D}^{2}(S x, S p) \leq\|x-p\|^{2}+k d^{2}(x, S x) \text {. }
$$

If $k=1 \mathrm{in}(7), S$ is hemicontractive in the terminology of Naimpally and Singh [7] for single-valued hemicontractive, while $S$ is quasi-nonexpansive if $k=0$.

Furthermore, every multivalued $k$-strictly pseudocontractive-type in the sense of [1] with the nonempty set of strict fixed points is demicontractive with respect to its set of strict fixed points.

A single-valued mapping $S: D(S) \subseteq H \longrightarrow H$ is called nonspreading in the sense of Kohsaka and Takahashi $[8,9]$ if

$$
\|S x-S y\|^{2} \leq\|x-y\|^{2}+2\langle x-S x, y-S y\rangle, \quad \forall x, y \in C .
$$

Observe that if $S$ is nonspreading and $F(S) \neq \varnothing$, then $S$ is quasi-nonexpansive. $S$ is $k$-strictly pseudo-nonspreading in the sense of Osilike and Isiogugu [10] if there exists $k \in[0,1)$ such that

$$
\begin{gathered}
\|S x-S y\|^{2} \leq\|x-y\|^{2}+k\|x-S x-(y-S y)\|^{2} \\
+2\langle x-S x, y-S y\rangle
\end{gathered}
$$

for all $x, y \in D(S)$. Clearly, every nonspreading mapping is $k$-strictly pseudo-nonspreading. If $S$ is $k$-strictly pseudononspreading and $F(S) \neq \varnothing$, then $S$ is demicontractive in the sense of [6] (see also [11]).

Several algorithms have been introduced by different authors for the approximation of common fixed points of finite family of mappings $\left\{S_{i}\right\}_{i=1}^{N}$, where $N \in \mathbb{N}$ (the set of nonnegative integers) (see, for example, [12-18] and references therein). One of the motivations for this aspect of research is the well-known convex feasibility problem which is reducible to the problem of finding a point in the intersection of the set of fixed points of a family of nonexpansive mappings (see, for example, $[19,20]$ ). The earliest of such algorithms was the cyclic algorithm introduced by Bauschke [12] using a Halpern-type iterative process considered in [21] for the approximation of a common fixed point of a finite family of nonexpansive self-mappings. $\mathrm{He}$ proved the following theorem.

Theorem 1 (see [12], Theorem 3.1). Let $K$ be a nonempty convex closed subset of a real Hilbert space $H$ and $S_{1}, S_{2}, \ldots, S_{N}$ be a finite family of nonexpansive mappings of $K$ into itself with $F:=\cap_{i=1}^{N} F\left(S_{i}\right) \neq \varnothing$ with $F=$
$F\left(S_{N} S_{N-1} \ldots S_{1}\right)=F\left(S_{1} S_{N} \ldots S_{2}\right)=F\left(S_{N-1} S_{N-2} \ldots S_{1} S_{N}\right)$. Given points $u, x_{0} \in K$, let $\left\{x_{n}\right\}$ be generated by

$$
x_{n+1}=\varsigma_{n} x_{n}+\left(1-\varsigma_{n}\right) S_{n+1} x_{n+1}, \quad n \geq 0,
$$

where $S_{n}:=S_{n(\bmod N)}$ and $\varsigma_{n} \subset(0,1)$ satisfies $\sum_{n \geq 1}\left|\varsigma_{n+N}-\varsigma_{n}\right|$ $<\infty$. Then, $\left\{x_{n}\right\}$ converges strongly to $P_{F} u$, where $P_{F}: H \longrightarrow$ $F$ is the metric projection.

The above algorithm of Bauschke was extended to approximate the family of more general class of strictly pseudocontractive mappings (see, for example, [22, 23]). Suantai et al. also considered similar algorithms (see, for example, [24]) and references therein.

In 2008, Zhang and Guo [25] considered a parallel iteration for approximating the common fixed points of a finite family of strictly pseudo-contractive mapping. They obtained the following theorem.

Theorem 2 (see [25], Theorem 4.3). Let $E$ be a real $q$ uniformly smooth Banach space which is also uniformly convex and $K$ be a nonempty convex closed subset of $E$. Let $N \geq 1$ be an integer, and for each $1 \leq i \leq N$, let $S_{i}: K \longrightarrow K$ be a $k_{i}$-strictly pseudo-contractive mapping for some $0 \leq k_{i}<1$. Let $k=\min \left\{k_{i}: 1 \leq i \leq N\right\}$. Assume the common fixed point set $\cap_{i-1}^{N} F\left(S_{i}\right)$ is nonempty. Assume also for each $n,\left\{\lambda_{i}^{n}\right\}_{i=1}^{N}$ is a finite sequence of positive numbers such that $\sum_{1=1}^{N} \lambda_{i}^{n}=1$ for all $n$ and $\inf _{n \geq 1} \lambda_{i}^{n}>0$ for all $1 \leq i \leq N$. Given $x_{0} \in K$, let $\left\{x_{n}\right\}_{n=1}^{\infty}$ be the sequence generated by the algorithm:

$$
x_{n+1}=\varsigma_{n} x_{n}+\left(1-\varsigma_{n}\right) \sum_{i=1}^{N} \lambda_{i}^{n} S_{i} x_{n}, \quad n \geq 0 .
$$

Let $\left\{\varsigma_{n}\right\}_{n=1}^{\infty}$ be a real sequence satisfying the conditions

$$
\begin{array}{r}
\sum_{n=0}^{\infty} \sum_{i=1}^{N}\left|\lambda_{i}^{n+1}-\lambda_{i}^{n}\right|<\infty \\
\sum_{n=0}^{\infty}\left(1-\varsigma_{n}\right) q k-\left[C q\left(1-\varsigma_{n}\right)^{q-1}\right]=\infty .
\end{array}
$$
$\left\{S_{i}\right\}_{i=1}^{N}$.

Then, $\left\{x_{n}\right\}$ converges weakly to a common fixed point of

Motivated by the parallel algorithm above, many authors have considered in a real Hilbert space, another form of parallel algorithm for a finite family $\left\{S_{i}\right\}_{i=1}^{N}$ of $k_{i}$-strictly pseudo-contractive mappings defined by

$$
x_{n+1}=\varsigma_{n}^{0} x_{n}+\sum_{i=1}^{N} \varsigma_{n}^{i} S_{i} x_{n}, \quad n \geq 1,
$$

where $\left\{\varsigma_{n}^{i}\right\}_{n=1}^{\infty} \subseteq(0,1)$ for each $i$ and $\sum_{i=0}^{N} \varsigma_{n}^{i}=1$ for each $n$ (see, for example, [13] and references therein).

In [14], Iemoto and Takahashi studied the approximation of common fixed points of a nonexpansive self-mapping $T$ and a nonspreading self-mapping $S$ in a real Hilbert space. If $T, S: C \longrightarrow C$ are, respectively, nonexpansive and nonspreading mappings, they considered the iterative scheme $\left\{x_{n}\right\}_{n=1}^{\infty}$ generated from arbitrary $x_{1} \in C$ by 


$$
x_{n+1}=\left(1-\varsigma_{n}\right) x_{n}+\varsigma_{n}\left[\beta_{n} S x_{n}+\left(1-\beta_{n}\right) T x_{n}\right], \quad n \geq 1,
$$

where $\left\{c_{n}\right\}$ and $\left\{\beta_{n}\right\}$ are suitable sequences in $[0,1]$. They proved the following main theorem:

Theorem 3 (see [14], Theorem 4.1). Let $H$ be a real Hilbert space. Let $C$ be a nonempty convex and closed subset of $H$. Let $S$ be a nonspreading mapping of $C$ into itself and $T$ a nonexpansive mapping of $C$ into itself such that $F(T) \cap F(S) \neq \varnothing$. Define a sequence $\left\{x_{n}\right\}_{n=1}^{\infty}$ in $C$ as follows:

$$
\left\{\begin{array}{l}
x_{1} \in C, \\
x_{n+1}=\left(1-\varsigma_{n}\right) x_{n}+\varsigma_{n}\left[\beta_{n} S x_{n}+\left(1-\beta_{n}\right) T x_{n}\right],
\end{array}\right.
$$

for all $n \in \mathbb{N}$, where $\left\{\varsigma_{n}\right\}_{n=1}^{\infty},\left\{\beta_{n}\right\}_{n=1}^{\infty} \subset[0,1]$.

Then, the following hold:

(i) If $\liminf _{n \longrightarrow \infty} \varsigma_{n}\left(1-\varsigma_{n}\right)>0, \quad \sum_{n=1}^{\infty}\left(1-\beta_{n}\right)<\infty$, then $\left\{x_{n}\right\}_{n=1}^{\infty}$ converges weakly to $v \in F(S)$.

(ii) If $\sum_{n=1}^{\infty} \varsigma_{n}\left(1-\varsigma_{n}\right)=\infty$ and $\sum_{n=1}^{\infty} \beta_{n}<\infty$, then $\left\{x_{n}\right\}_{n=1}^{\infty}$ converges weakly to $v \in F(T)$.

(iii) If $\liminf _{n \longrightarrow \infty} \varsigma_{n}\left(1-\varsigma_{n}\right)>0$ and $\liminf _{n \longrightarrow \infty} \beta_{n}$ $\left(1-\beta_{n}\right)>0$, then $\left\{x_{n}\right\}_{n=1}^{\infty}$ converges weakly to $v \in F(T) \cap F(S)$.

Motivated by the above result, Osilike and Isiogugu obtained the following result.

Theorem 4 (see [10], Theorem 3.1.1). Let $C$ be a nonempty convex closed subset of a real Hilbert space, and let $T: C \longrightarrow C$ be a k-strictly pseudo-nonspreading mapping with a nonempty fixed point set $F(T)$. Let $\beta \in[k, 1)$, and let $\left\{\varsigma_{n}\right\}_{n=1}^{\infty}$ be a real sequence in $[0,1)$ such that $\lim _{n \longrightarrow \infty} \varsigma_{n}=0$. Let $\left\{x_{n}\right\}_{n=1}^{\infty}$ and $\left\{z_{n}\right\}_{n=1}^{\infty}$ be sequences in $C$ generated for arbitrary $x_{1} \in C$ by

$$
\begin{aligned}
x_{n+1} & =\varsigma_{n} x_{n}+\left(1-\varsigma_{n}\right)\left[\beta x_{n}+(1-\beta) T x_{n}\right], \quad n \geq 1, \\
z_{n} & =\frac{1}{n} \sum_{k=1}^{n} x_{k}, \quad n \geq 1 .
\end{aligned}
$$

Then, $\left\{z_{n}\right\}_{n=1}^{\infty}$ converges weakly to $z \in F(T)$, where $z=\lim _{n \longrightarrow \infty} P_{F(T)} x_{n}$.

We observed that all the existing iteration schemes for the approximation of a common fixed point of a finite family $\left\{T_{1}, T_{2}, \ldots, T_{N}\right\}$ of mappings for $N>2$, which are related to the parallel algorithm, require the condition that, for each $n$, $\sum_{i=1}^{N} \zeta_{n}^{i}=1$ on the control sequences $\left\{\left\{c_{n}^{i}\right\}_{n=1}^{\infty}\right\}_{i=1}^{N}$. However, in real-life applications, if $N$ is very large, it is very difficult or almost impossible to generate a family of such control sequences. Moreover, the computational cost of generating such a family of control sequences is very high and also takes a very long process. On the contrary, the algorithms of Iemoto and Takahashi [14] and Osilike and Isiogugu [10] do not require the imposition $\sum_{i=1}^{N} \zeta_{n}^{i}=1$ on the control sequences for $N=2$. Consequently, there is a need to extend the iteration schemes in $[10,14]$ for $N>2$.
Motivated by the above observations and the algorithms of Iemoto and Takahashi [14] and Osilike and Isiogugu [10], which do not require the imposition $\sum_{i=1}^{N} \zeta_{n}^{i}=1$ on the control sequences for $N=2$ and the need to extend the iteration schemes for $N>2$, the aim of this work is first to study some possible linear combinations of the products of the elements of a family of sequence of real numbers $\left\{\left\{\varsigma_{n}^{i}\right\}_{n=1}^{\infty}\right\}_{i=1}^{N}$ whose sum is unity. Second, to apply the result to construct a new (horizontal) algorithm which does not require the condition $\sum_{i=1}^{N} \zeta_{n}^{i}=1$ on the finite family of the control sequences $\left\{\left\{c_{n}^{i}\right\}_{n=1}^{\infty}\right\}_{i=1}^{N}$. Third, to prove that the new algorithm converges weakly and strongly to an element in the intersection of the set of fixed points of a countable finite family of multivalued type-one demicontractive mappings. We also show that our algorithm is an extension of the algorithm of Osilike and Isiogugu [10] when $N=2$. Furthermore, the algorithm is applied to establish some new algorithms for the approximation of the common solution of an equilibrium problem and a fixed point problem for a finite family of type demicontractive mappings. The numerical examples and computations of the horizontal algorithm were also presented. The obtained results complement, extend, and improve many results on the iteration schemes for the approximation of common fixed points for a finite family of single-valued and multivalued mappings.

\section{Preliminaries}

In the sequel, we shall need the following definitions and lemmas.

Definition 1 (see, e.g., [26-27]). Let $Y$ be a Banach space and $S: D(S) \subseteq Y \longrightarrow 2^{Y}$ be a multivalued mapping. $I-S$ is weakly demiclosed at zero if for any sequence, $\left\{x_{n}\right\}_{n=1}^{\infty} \subseteq D(S)$ such that $\left\{x_{n}\right\}$ converges weakly to $p$ and a sequence $\left\{y_{n}\right\}$ with $y_{n} \in S x_{n}$ for all $n \in \mathbb{N}$ such that $\left\{x_{n}-y_{n}\right\}$ strongly converges to zero. Then, $p \in S p$ (i.e., $0 \in(I-S) p$ ).

Definition 2. A Banach space $Y$ is said to satisfy Opial's condition [28] if whenever a sequence $\left\{x_{n}\right\}$ weakly converges to $x \in Y$, then it is the case that

$$
\lim \inf \left\|x_{n}-x\right\|<\lim \inf \left\|x_{n}-y\right\|,
$$

for all $y \in Y, y \neq x$.

Definition 3 (see [29]). A multivalued mapping $S: C \longrightarrow P(C)$ is said to satisfy condition (1) (see, for example, [29]) if there exists a nondecreasing function $f:[0, \infty) \longrightarrow[0, \infty)$ with $f(0)=0$ and $f(r)>0$ for all $r \in(0, \infty)$ such that

$$
d(x, S x) \geq f(d(x, F(S))), \quad \forall x \in C .
$$

Definition 4 (see [4]). Let $Y$ be a normed space and $S: D(S) \subseteq Y \longrightarrow 2^{Y}$ be a multivalued map. $S$ is of type-one if given any pair $x, y \in D(S)$, then

$$
\|u-v\| \leq \mathscr{D}(S x, S y), \quad \text { for all } u \in P_{S} x, v \in P_{S} y .
$$


Lemma 1 (see [30]). Let $\left\{a_{n}\right\}$ and $\left\{\gamma_{n}\right\}$ be sequences of nonnegative real numbers satisfying the following relation:

$$
a_{n+1} \leq a_{n}+\gamma_{n}, \quad \forall n \in N .
$$

If $\sum \gamma_{n}<\infty$, then $\lim _{n \rightarrow \infty} a_{n}$ exists.

\section{Main Results}

Let $K$ be a nonempty convex and closed subset of a real Hilbert space $H$. Suppose that $\left\{S_{i}\right\}_{i=1}^{N}, N \geq 2$ is a countable finite family of mappings $S_{i}: K \longrightarrow K$, and we consider the horizontal iteration process generated from arbitrary $x_{1}$ for the finite family of mappings $\left\{S^{i}\right\}_{i=1}^{N}$, using a finite family of the control sequences $\left\{\left\{\varsigma_{n}^{i}\right\}_{n=1}^{\infty}\right\}_{i=1}^{N}$ as follows:

For $N=2$,

$$
x_{n+1}=\varsigma_{n}^{1} x_{n}+\left(1-\varsigma_{n}^{1}\right)\left[\varsigma_{n}^{2} S_{1} x_{n}+\left(1-\varsigma_{n}^{2}\right) S_{2} x_{n}\right] .
$$

For $N=3$,

$$
\begin{aligned}
x_{n+1}= & \varsigma_{n}^{1} x_{n}+\left(1-\varsigma_{n}^{1}\right)\left[\varsigma_{n}^{2} S_{1} x_{n}+\left(1-\varsigma_{n}^{2}\right)\right. \\
& \left.\cdot\left[\varsigma_{n}^{3} S_{2} x_{n}+\left(1-\varsigma_{n}^{3}\right) S_{3} x_{n}\right]\right] .
\end{aligned}
$$

For arbitrary but finite $N \geq 2$,

$$
\begin{aligned}
x_{n+1}= & \varsigma_{n}^{1} x_{n}+\left(1-\varsigma_{n}^{1}\right)\left[\varsigma_{n}^{2} S_{1} x_{n}+\left(1-\varsigma_{n}^{2}\right)\left[\varsigma_{n}^{3} S_{2} x_{n}\right.\right. \\
& \left.\left.+\left(1-\varsigma_{n}^{3}\right)\left[\ldots\left[\varsigma_{N} S_{N-1} x_{n}+\left(1-\varsigma_{N}\right) S_{N} x_{n}\right] \ldots\right]\right]\right] \\
= & \varsigma_{n}^{1} x_{n}+\sum_{i=2}^{N} \varsigma_{n}^{i} \prod_{j=1}^{i-1}\left(1-\varsigma_{n}^{j}\right) S_{i-1} x_{n} \\
& +\prod_{j=1}^{N}\left(1-\varsigma_{n}^{j}\right) S_{N} x_{n}, n \geq 1 .
\end{aligned}
$$

We now present the following results which are very useful in establishing our convergence theorems.

Proposition 1. Let $\left\{c_{i}\right\}_{i=1}^{N} \subseteq \mathbb{R}$ be a countable subset of the set of real numbers $\mathbb{R}$, where $N \geq 2$ is an arbitrary integer. Then, the following holds:

$$
\varsigma_{1}+\sum_{i=2}^{N} \varsigma_{i} \prod_{j=1}^{i-1}\left(1-\varsigma_{j}\right)+\prod_{j=1}^{N}\left(1-\varsigma_{j}\right)=1 .
$$

Proof. For $N=2$,

$$
\begin{aligned}
\varsigma_{1} & +\sum_{i=2}^{2} \varsigma_{i} \prod_{j=1}^{i-1}\left(1-\varsigma_{j}\right)+\prod_{j=1}^{2}\left(1-\varsigma_{j}\right) \\
& =\varsigma_{1}+\varsigma_{2}\left(1-\varsigma_{1}\right)+\left(1-\varsigma_{1}\right)\left(1-\varsigma_{2}\right) \\
& =\varsigma_{1}+\left(1-\varsigma_{1}\right)\left[\varsigma_{2}+\left(1-\varsigma_{2}\right)\right] \\
& =\varsigma_{1}+\left(1-\varsigma_{1}\right)=1 .
\end{aligned}
$$

We assume it is true for $N$ and prove for $N+1$.

$$
\begin{aligned}
\varsigma_{1} & +\sum_{i=2}^{N+1} \varsigma_{i} \prod_{j=1}^{i-1}\left(1-\varsigma_{j}\right)+\prod_{j=1}^{N+1}\left(1-\varsigma_{j}\right) \\
& =\varsigma_{1}+\sum_{i=2}^{N} \varsigma_{i} \prod_{j=1}^{i-1}\left(1-\varsigma_{j}\right)+\varsigma_{N+1} \prod_{j=1}^{N}\left(1-\varsigma_{j}\right)+\prod_{j=1}^{N+1}\left(1-\varsigma_{j}\right) \\
& =\varsigma_{1}+\sum_{i=2}^{N} \varsigma_{i} \prod_{j=1}^{i-1}\left(1-\varsigma_{j}\right)+\prod_{j=1}^{N}\left(1-\varsigma_{j}\right)\left[\varsigma_{N+1}+\left(1-\varsigma_{N+1}\right)\right] \\
& =\varsigma_{1}+\sum_{i=2}^{N} \varsigma_{i} \prod_{j=1}^{i-1}\left(1-\varsigma_{j}\right)+\prod_{j=1}^{N}\left(1-\varsigma_{j}\right)=1 .
\end{aligned}
$$

Remark 1. Proposition 1 holds if $\left\{\varsigma_{i}\right\}_{i=1}^{N}$ is replaced with $\left\{c_{i}\right\}_{i=0}^{N}$.

Proposition 2. Let $\left\{\varsigma_{i}\right\}_{i=k}^{N} \subseteq \mathbb{R}$ be a countable subset of the set of real numbers $\mathbb{R}$, where $k$ is a fixed nonnegative integer and $N \in \mathbb{N}$ is any integer with $k+1 \leq N$. Then, the following holds:

$$
\varsigma_{k}+\sum_{i=k+1}^{N} \varsigma_{i} \prod_{j=k}^{i-1}\left(1-\varsigma_{j}\right)+\prod_{j=k}^{N}\left(1-\varsigma_{j}\right)=1 .
$$

Proof. For $k=0, N=1$, and $k=1, N=2$, the proofs follow from Remark 1 and Proposition 1, respectively.

We assume it is true for $k$ and $N$. Now, for $k$ and $N+1$,

$$
\begin{aligned}
\varsigma_{k} & +\sum_{i=k+1}^{N+1} \varsigma_{i} \prod_{j=k}^{i-1}\left(1-\varsigma_{j}\right)+\prod_{j=k}^{N+1}\left(1-\varsigma_{j}\right) \\
= & \varsigma_{k}+\sum_{i=k+1}^{N} \varsigma_{i} \prod_{j=k}^{i-1}\left(1-\varsigma_{j}\right)+\varsigma_{N+1} \prod_{j=k}^{N}\left(1-\varsigma_{j}\right)+\prod_{j=k}^{N+1}\left(1-\varsigma_{j}\right) \\
= & \varsigma_{k}+\sum_{i=k+1}^{N} \varsigma_{i} \prod_{j=k}^{i-1}\left(1-\varsigma_{j}\right)+\prod_{j=k}^{N}\left(1-\varsigma_{j}\right)\left[\varsigma_{N+1}+\left(1-\varsigma_{N+1}\right)\right] \\
= & \varsigma_{k}+\sum_{i=k+1}^{N} \varsigma_{i} \prod_{j=k}^{i-1}\left(1-\varsigma_{j}\right)+\prod_{j=k}^{N}\left(1-\varsigma_{j}\right) \\
= & \varsigma_{k}+\sum_{i=k}^{N} \varsigma_{i} \prod_{j=k}^{i-1}\left(1-\varsigma_{j}\right)+\prod_{j=k}^{N}\left(1-\varsigma_{j}\right)=1 .
\end{aligned}
$$

Proposition 3. Let $t, u$, and $v$ be arbitrary elements of a real Hilbert space $H$. Let $k$ be a fixed nonnegative integer and $N \in \mathbb{N}$ be such that $k+1 \leq N$. Let $\left\{v_{i}\right\}_{i=k}^{N-1} \subseteq H$ and $\left\{c_{i}\right\}_{i=k}^{N} \subseteq[0,1]$ be a countable finite subset of $H$ and $\mathbb{R}$, respectively. Define

$$
y=\varsigma_{k} t+\sum_{i=k+1}^{N} \varsigma_{i} \prod_{j=k}^{i-1}\left(1-\varsigma_{j}\right) v_{i-1}+\prod_{j=k}^{N}\left(1-\varsigma_{j}\right) v .
$$




$$
\begin{aligned}
\|y-u\|^{2}= & \varsigma_{k}\|t-u\|^{2}+\sum_{i=k+1}^{N} \varsigma_{i} \prod_{j=k}^{i-1}\left(1-\varsigma_{j}\right)\left\|v_{i-1}-u\right\|^{2}+\prod_{j=k}^{N}\left(1-\varsigma_{j}\right)\|v-u\|^{2} \\
& -\varsigma_{k}\left[\sum_{i=k+1}^{N} \varsigma_{i} \prod_{j=k}^{i-1}\left(1-\varsigma_{j}\right)\left\|t-v_{i-1}\right\|^{2}+\prod_{j=k}^{N}\left(1-\varsigma_{j}\right)\|t-v\|^{2}\right] \\
& -\left(1-\varsigma_{k}\right)\left[\sum_{i=k+1}^{N-1} \varsigma_{i} \prod_{j=k}^{i}\left(1-\varsigma_{j}\right)\left\|v_{i-1}-\left[\varsigma_{i+1} v_{i}+w_{i+1}\right]\right\|^{2}\right. \\
& \left.+\varsigma_{N} \prod_{j=k}^{N}\left(1-\varsigma_{j}\right)\left\|v-v_{N-1}\right\|^{2}\right],
\end{aligned}
$$

where $w_{k}=\sum_{i=k+1}^{N} \varsigma_{i} \prod_{j=k}^{i-1}\left(1-\varsigma_{j}\right) v_{i-1}+\prod_{j=k}^{N}\left(1-\varsigma_{j}\right) v, \quad k=$ $1,2, \ldots, N-1$, and $w_{N}=\left(1-\varsigma_{N}\right) v$.

Proof. Using the well-known identity, $\|t x+(1-t) y\|^{2}=t\|x\|^{2}+(1-t)\|y\|^{2}-t(1-t)\|x-y\|^{2}$, which holds for all $x, y \in H$ and for all $t \in[0,1]$, we prove by (i) direct computation and (ii) induction.

Observe that, for $k \leq N-1, \quad w_{k}=\left(1-\varsigma_{k}\right)$ $\left[\varsigma_{k+1} v_{k}+w_{k+1}\right]$. Consequently, by the direct computation, we have

$$
\begin{aligned}
\|y-u\|^{2}= & \left\|\varsigma_{k} t+\sum_{i=k+1}^{N} \varsigma_{i} \prod_{j=k}^{i-1}\left(1-\varsigma_{j}\right) v_{i-1}+\prod_{j=k}^{N}\left(1-\varsigma_{j}\right) v-u\right\|^{2} \\
= & \left\|\varsigma_{k} t+w_{k}-u\right\|^{2} \\
= & \left.\| \varsigma_{k} t+\left(1-\varsigma_{k}\right)\left[\varsigma_{k+1} v_{k}+w_{k+1}\right]-u\right] \|^{2} \\
= & \varsigma_{k}\|t-u\|^{2}+\left(1-\varsigma_{k}\right)\left\|\varsigma_{k+1} v_{k}+w_{k+1}-u\right\|^{2}-\varsigma_{k}\left(1-\varsigma_{k}\right)\left\|t-\left[\varsigma_{k+1} v_{k}+w_{k+1}\right]\right\|^{2} \\
= & \varsigma_{k}\|t-u\|^{2}+\left(1-\varsigma_{k}\right)\left[\varsigma_{k+1}\left\|v_{k}-u\right\|^{2}+\left(1-\varsigma_{k+1}\right)\left\|\varsigma_{k+2} v_{k+1}+w_{k+2}-u\right\|^{2}\right. \\
& \left.-\varsigma_{k+1}\left(1-\varsigma_{k+1}\right)\left\|v_{k}-\left[\varsigma_{k+2} v_{k+1}+w_{k+2}\right]\right\|^{2}\right] \\
& -\varsigma_{k}\left(1-\varsigma_{k}\right)\left[\varsigma_{k+1}\left\|t-v_{k}\right\|^{2}+\left(1-\varsigma_{k+1}\right)\left\|t-\left[\varsigma_{k+2} v_{k+1}+w_{k+2}\right]\right\|^{2}\right. \\
& \left.-\varsigma_{k+1}\left(1-\varsigma_{k+1}\right)\left\|v_{k}-\left[\varsigma_{k+2} v_{k+1}+w_{k+2}\right]\right\|^{2}\right] \\
= & \varsigma_{k}\|t-u\|^{2}+\left(1-\varsigma_{k}\right) \varsigma_{k+1}\left\|v_{k}-u\right\|^{2} \\
& +\left(1-\varsigma_{k}\right)\left(1-\varsigma_{k+1}\right)\left\|\varsigma_{k+2} v_{k+1}+w_{k+2}-u\right\|^{2} \\
& -\left(1-\varsigma_{k}\right) \varsigma_{k+1}\left(1-\varsigma_{k+1}\right)\left\|v_{k}-\left[\varsigma_{k+2} v_{k+1}+w_{k+2}\right]\right\|^{2} \\
& -\varsigma_{k}\left(1-\varsigma_{k}\right) \varsigma_{k+1}\left\|t-v_{k}\right\|^{2}-\varsigma_{k}\left(1-\varsigma_{k}\right)\left(1-\varsigma_{k+1}\right)\left\|t-\left[\varsigma_{k+2} v_{k+1}+w_{k+2}\right]\right\|^{2} \\
& \left.+\varsigma_{k}\left(1-\varsigma_{k}\right) \varsigma_{k+1}\left(1-\varsigma_{k+1}\right)\left\|v_{k}-\left[\varsigma_{k+2} v_{k+1}+w_{k+2}\right]\right\|^{2}\right] \\
& +u\left\|^{2}+\left(1-\varsigma_{k}\right) \varsigma_{k+1}\right\| v_{k}-u\left\|^{2}-\varsigma_{k}\left(1-\varsigma_{k}\right) \varsigma_{k+1}\right\| t-v_{k} \|^{2} \\
& +\left(1-\varsigma_{k+1}\right)\left\|\varsigma_{k+2} v_{k+1}+w_{k+2}-u\right\|^{2} \\
& \left(1-\varsigma_{k+1}\right)\left\|t-\left[\varsigma_{k+2} v_{k+1}+w_{k+2}\right]\right\|^{2} \\
& \left(1-\varsigma_{k}\right)\left\|v_{k}-\left[\varsigma_{k+2} v_{k+1}+w_{k+2}\right]\right\|^{2}
\end{aligned}
$$




$$
\begin{aligned}
& =\varsigma_{k}\|t-u\|^{2}+\left(1-\varsigma_{k}\right) \varsigma_{k+1}\left\|v_{k}-u\right\|^{2}-\varsigma_{k}\left(1-\varsigma_{k}\right) \varsigma_{k+1}\left\|t-v_{k}\right\|^{2} \\
& -\varsigma_{k+1}\left(1-\varsigma_{k}\right)\left(1-\varsigma_{k+1}\right)\left(1-\varsigma_{k}\right)\left\|v_{k}-\left[\varsigma_{k+2} v_{k+1}+w_{k+2}\right]\right\|^{2} \\
& +\left(1-\varsigma_{k}\right)\left(1-\varsigma_{k+1}\right)\left\|\varsigma_{k+2} v_{k+1}+w_{k+2}-u\right\|^{2} \\
& -\varsigma_{k}\left(1-\varsigma_{k}\right)\left(1-\varsigma_{k+1}\right)\left\|t-\left[\varsigma_{k+2} v_{k+1}+w_{k+2}\right]\right\|^{2} \\
& =\varsigma_{k}\|t-u\|^{2}+\left(1-\varsigma_{k}\right) \varsigma_{k+1}\left\|v_{k}-u\right\|^{2}-\varsigma_{k}\left(1-\varsigma_{k}\right) \varsigma_{k+1}\left\|t-v_{k}\right\|^{2} \\
& -\varsigma_{k+1}\left(1-\varsigma_{k}\right)\left(1-\varsigma_{k+1}\right)\left(1-\varsigma_{k}\right)\left\|v_{k}-\left[\varsigma_{k+2} v_{k+1}+w_{k+2}\right]\right\|^{2} \\
& +\left(1-\varsigma_{k}\right)\left(1-\varsigma_{k+1}\right)\left\|\varsigma_{k+2} v_{k+1}+\left(1-\varsigma_{k+2}\right)\left[\varsigma_{k+3} v_{k+2}+w_{k+3}\right]-u\right\|^{2} \\
& -\varsigma_{k}\left(1-\varsigma_{k}\right)\left(1-\varsigma_{k+1}\right)\left\|\varsigma_{k+2} v_{k+1}+\left(1-\varsigma_{k+2}\right)\left[\varsigma_{k+3} v_{k+2}+w_{k+3}\right]-t\right\|^{2} \\
& =\varsigma_{k}\|t-u\|^{2}+\left(1-\varsigma_{n}^{1}\right) \varsigma_{k+1}\left\|v_{k}-u\right\|^{2}-\varsigma_{n}^{1}\left(1-\varsigma_{n}^{1}\right) \varsigma_{k+1}\left\|t-v_{k}\right\|^{2} \\
& +\left(1-\varsigma_{k}\right)\left(1-\varsigma_{k+1}\right) \varsigma_{k+2}\left\|v_{k+1}-u\right\|^{2} \\
& +\left(1-\varsigma_{k}\right)\left(1-\varsigma_{k+1}\right)\left(1-\varsigma_{k+2}\right)\left\|\varsigma_{k+3} v_{k+2}+w_{k+3}-u\right\|^{2} \\
& -\left(1-\varsigma_{k}\right)\left(1-\varsigma_{k+1}\right) \varsigma_{k+2}\left(1-\varsigma_{k+2}\right)\left\|v_{k+1}-\left[\varsigma_{k+3} v_{k+2}+w_{k+3}\right]\right\|^{2} \\
& -\varsigma_{k}\left(1-\varsigma_{k}\right)\left(1-\varsigma_{k+1}\right) \varsigma_{k+2}\left\|v_{k+1}-t\right\|^{2} \\
& -\varsigma_{k}\left(1-\varsigma_{k}\right)\left(1-\varsigma_{k+1}\right)\left(1-\varsigma_{k+2}\right)\left\|\varsigma_{k+3} v_{k+2}+w_{k+3}-t\right\|^{2} \\
& +\varsigma_{k}\left(1-\varsigma_{k}\right)\left(1-\varsigma_{k+1}\right) \varsigma_{k+2}\left(1-\varsigma_{k+2}\left\|v_{k+1}-\varsigma_{k+3} v_{k+2}+w_{k+3}\right\|^{2}\right. \\
& -\varsigma_{k+1}\left(1-\varsigma_{k}\right)\left(1-\varsigma_{k+1}\right)\left(1-\varsigma_{k}\right)\left\|v_{k}-\left[\varsigma_{k+2} v_{k+1}+w_{k+2}\right]\right\|^{2} \\
& =\varsigma_{k}\|t-u\|^{2}+\left(1-\varsigma_{k}\right) \varsigma_{k+1}\left\|v_{k}-u\right\|^{2}-\varsigma_{k}\left(1-\varsigma_{k}\right) \varsigma_{k+1}\left\|t-v_{k}\right\|^{2} \\
& +\left(1-\varsigma_{k}\right)\left(1-\varsigma_{k+1}\right) \varsigma_{k+2}\left\|v_{k+1}-u\right\|^{2} \\
& +\left(1-\varsigma_{k}\right)\left(1-\varsigma_{k+1}\right)\left(1-\varsigma_{k+2}\right)\left\|\varsigma_{k+3} v_{k+2}+w_{k+3}-u\right\|^{2} \\
& -\varsigma_{k}\left(1-\varsigma_{k}\right)\left(1-\varsigma_{k+1}\right) \varsigma_{k+2}\left\|v_{k+1}-t\right\|^{2} \\
& -\varsigma_{k}\left(1-\varsigma_{k}\right)\left(1-\varsigma_{k+1}\right)\left(1-\varsigma_{k+2}\right)\left\|\varsigma_{k+3} v_{k+2}+w_{k+3}-t\right\|^{2} \\
& -\varsigma_{k+1}\left(1-\varsigma_{k}\right)\left(1-\varsigma_{k+1}\right)\left(1-\varsigma_{k}\right)\left\|v_{k}-\left[\varsigma_{k+2} v_{k+1}+w_{k+2}\right]\right\|^{2} \\
& -\left(1-\varsigma_{k}\right)^{2}\left(1-\varsigma_{k+1}\right) \varsigma_{k+2}\left(1-\varsigma_{k+2}\right)\left\|v_{k+1}-\left[\varsigma_{k+3} v_{k+2}+w_{k+3}\right]\right\|^{2} \text {, } \\
& =\varsigma_{k}\|t-u\|^{2}+\sum_{i=k+1}^{k+2} \varsigma_{i} \prod_{j=k}^{i-1}\left(1-\varsigma_{j}\right)\left\|v_{i-1}-u\right\|^{2} \\
& -\varsigma_{k}\left[\sum_{i=k+1}^{k+2} \varsigma_{i} \prod_{j=k}^{i-1}\left(1-\varsigma_{j}\right)\left\|t-v_{i-1}\right\|^{2}\right] \\
& -\left(1-\varsigma_{k}\right)\left[\sum_{i=k+1}^{k+2} \varsigma_{i} \prod_{j=k}^{i}\left(1-\varsigma_{j}\right)\left\|v_{i-1}-\left[\varsigma_{i+1} v_{i}+w_{i+1}\right]\right\|^{2}\right. \\
& +\prod_{j=k}^{k+2}\left(1-\varsigma_{j}\right)\left\|\left[\varsigma_{k+3} v_{k+2}+w_{k+3}\right]-u\right\|^{2} \\
& -\varsigma_{k} \prod_{j=k}^{k+2}\left(1-\varsigma_{j}\right)\left\|t-\left[\varsigma_{k+3} v_{k+2}+w_{k+3}\right]\right\|^{2} \\
& = \\
& \text { ! }
\end{aligned}
$$




$$
\begin{aligned}
= & \varsigma_{k}\|t-u\|^{2}+\sum_{i=k+1}^{N} \varsigma_{i} \prod_{j=k}^{i-1}\left(1-\varsigma_{j}\right)\left\|v_{i-1}-u\right\|^{2}+\prod_{j=k}^{N}\left(1-\varsigma_{j}\right)\|v-u\|^{2} \\
& -\varsigma_{k}\left[\sum_{i=k+1}^{N} \varsigma_{i} \prod_{j=k}^{i-1}\left(1-\varsigma_{j}\right)\left\|t-v_{i-1}\right\|^{2}+\prod_{j=k}^{N}\left(1-\varsigma_{j}\right)\|t-v\|^{2}\right] \\
& -\left(1-\varsigma_{k}\right)\left[\sum_{i=k+1}^{N-1} \varsigma_{i} \prod_{j=k}^{i}\left(1-\varsigma_{j}\right)\left\|v_{i-1}-\left[\varsigma_{i+1} v_{i}+w_{i+1}\right]\right\|^{2}\right. \\
& \left.+\varsigma_{N} \prod_{j=k}^{N}\left(1-\varsigma_{j}\right)\left\|v-v_{N-1}\right\|^{2}\right] .
\end{aligned}
$$

Therefore, it holds for $k, N$ from direct computation.

Since induction holds for a fixed $k$ and each $N$ from direct computation, then it is true for $k, N=1,2,3$. Thus, to prove by induction, we then assume that it is true for $k, N$ and prove for $k$ and $N+1$. From

$$
y=\varsigma_{k} t+\sum_{i=k+1}^{N+1} \varsigma_{i} \prod_{j=k}^{i-1}\left(1-\varsigma_{j}\right) v_{i-1}+\prod_{j=k}^{N+1}\left(1-\varsigma_{j}\right) v,
$$

we have that

$$
\begin{aligned}
\|y-u\|^{2}= & \left\|\varsigma_{k} t+\sum_{i=k+1}^{N+1} \varsigma_{i} \prod_{j=k}^{i-1}\left(1-\varsigma_{j}\right) v_{i-1}+\prod_{j=k}^{N+1}\left(1-\varsigma_{j}\right) v-u\right\|^{2} \\
= & \left\|\varsigma_{k} t+\sum_{i=k+1}^{N} \varsigma_{i} \prod_{j=k}^{i-1}\left(1-\varsigma_{j}\right) v_{i-1}+\varsigma_{N+1} \prod_{j=k}^{N}\left(1-\varsigma_{j} j\right) v_{N}+\prod_{j=k}^{N+1}\left(1-\varsigma_{j}\right) v-u\right\|^{2} \\
= & \left\|\varsigma_{k} t+\sum_{i=k+1}^{N} \varsigma_{i} \prod_{j=k}^{i-1}\left(1-\varsigma_{j}\right) v_{i-1}+\prod_{j=k}^{N}\left(1-\varsigma_{j}\right)\left[\varsigma_{N+1} v_{N}+\left(1-\varsigma_{N+1}\right) v\right]-u\right\|^{2} \\
= & \left\|\varsigma_{k} t+\sum_{i=k+1}^{N} \varsigma_{i} \prod_{j=k}^{i-1}\left(1-\varsigma_{j}\right) v_{i-1}+\prod_{j=k}^{N}\left(1-\varsigma_{j}\right) v^{*}-u\right\|^{2} \\
= & \varsigma_{k}\|t-u\|^{2}+\sum_{i=k+1}^{N} \varsigma_{i} \prod_{j=k}^{i-1}\left(1-\varsigma_{j}\right)\left\|v_{i-1}-u\right\|^{2}+\prod_{j=k}^{N}\left(1-\varsigma_{j}\right)\left\|v^{*}-u\right\|^{2} \\
& +\varsigma_{k}\left[\sum_{i=k+1}^{N} \varsigma_{i} \prod_{j=k}^{i-k}\left(1-\varsigma_{j}\right)\left\|t-v_{i-1}\right\|^{2}+\prod_{j=k}^{N}\left(1-\varsigma_{j}\right)\left\|t-v^{*}\right\|^{2}\right] \\
& -\left(1-\varsigma_{k}\right)\left[\sum_{i=k+1}^{N-1} \varsigma_{i} \prod_{j=k}^{i}\left(1-\varsigma_{j}\right)\left\|v_{i-1}-\left[\varsigma_{i+1} v_{i}+w_{i+1}\right]\right\|^{2}\right. \\
& \\
& \left.\left\|v^{*}-v_{N-1}\right\|^{2}\right] .
\end{aligned}
$$

Observe that

$$
\begin{aligned}
\left\|t-v^{*}\right\|^{2}= & \left\|\varsigma_{N+1} v_{N}+\left(1-\varsigma_{N+1}\right) v-t\right\|^{2} \\
= & \varsigma_{N+1}\left\|t-v_{N}\right\|^{2}+\left(1-\varsigma_{N+1}\right)\|t-v\|^{2} \\
& -\varsigma_{N+1}\left(1-\varsigma_{N+1}\right)\left\|v_{N}-v\right\|^{2} .
\end{aligned}
$$

Also,

$$
\begin{aligned}
\left\|v^{*}-v_{N-1}\right\|^{2} & =\left\|\varsigma_{N+1} v_{N}+\left(1-\varsigma_{N+1}\right) v-v_{N-1}\right\|^{2} \\
& =\left\|v_{N-1}-\left[\varsigma_{N+1} v_{N}+\left(1-\varsigma_{N+1}\right) v\right]\right\|^{2} \\
& =\left\|v_{N-1}-\left[\varsigma_{N+1} v_{N}+w_{N+1}\right]\right\|^{2} .
\end{aligned}
$$


Furthermore,

$$
\begin{aligned}
\left\|v^{*}-u\right\|^{2}= & \left\|\varsigma_{N+1} v_{N}+\left(1-\varsigma_{N+1}\right) v-u\right\|^{2} \\
= & \varsigma_{N+1}\left\|u-v_{N}\right\|^{2}+\left(1-\varsigma_{N+1}\right)\|v-u\|^{2} \\
& -\varsigma_{N+1}\left(1-\varsigma_{N+1}\right)\left\|v_{N}-v\right\|^{2} .
\end{aligned}
$$

It then follows from (34-37) that

$$
\begin{aligned}
\|y-u\|^{2}= & \varsigma_{k}\|t-u\|^{2}+\sum_{i=k+1}^{N+1} \varsigma_{i} \prod_{j=k}^{i-1}\left(1-\varsigma_{j}\right)\left\|v_{i-1}-u\right\|^{2} \\
& +\prod_{j=k}^{N+1}\left(1-\varsigma_{j}\right)\|v-u\|^{2} \\
& -\varsigma_{k}\left[\sum_{i=k+1}^{N+1} \varsigma_{i} \prod_{j=k}^{i-i}\left(1-\varsigma_{j}\right)\left\|t-v_{i-1}\right\|^{2}+\prod_{j=k}^{N+1}\left(1-\varsigma_{j}\right)\|t-v\|^{2}\right] \\
& -\left(1-\varsigma_{k}\right)\left[\sum_{i=k+1}^{N} \varsigma_{i} \prod_{j=k}^{i}\left(1-\varsigma_{j}\right)\left\|v_{i-1}-\left[\varsigma_{i+1} v_{i}+w_{i+1}\right]\right\|^{2}\right. \\
& \left.+\varsigma_{N+1} \prod_{j=k}^{N+1}\left(1-\varsigma_{j}\right)\left\|v-v_{N}\right\|^{2}\right] .
\end{aligned}
$$

We now apply Propositions 2 and 3 to prove the following weak and strong convergence theorems for type-one demicontractive mappings.

Theorem 5. Let $K$ be a nonempty convex and closed subset of a real Hilbert space $H$. Suppose that $\left\{S_{i}\right\}_{i=1}^{N}, N \geq 2$ is a countable finite family of type-one demicontractive mappings $S_{i}: K \longrightarrow P(K)$ from $K$ into the family of all proximinal subsets of $K$ with contractive coefficients $\lambda_{i} \in[0,1)$ for each $i$. Suppose that $\cap{ }_{i=1}^{N} F\left(S_{i}\right) \neq \varnothing$ and for each $i,\left(I-S_{i}\right)$ is weakly demiclosed at zero; then, the sequence of the horizontal algorithm defined by

$$
\begin{aligned}
x_{n+1}= & \varsigma_{n, 1} x_{n}+\sum_{i=2}^{N} \varsigma_{n, i} \prod_{j=1}^{i-1}\left(1-\varsigma_{n, j}\right) y_{n, i-1} \\
& +\prod_{j=1}^{N}\left(1-\varsigma_{n, j}\right) y_{n, N}, \quad n \geq 1,
\end{aligned}
$$

converges weakly to $q \in \cap_{i=1}^{N} F\left(S_{i}\right)$, where $y_{n, i} \in S_{i} x_{n}$ for each $i$ and $\left\{\left\{\varsigma_{n, i}\right\}_{n=1}^{\infty}\right\}_{i=1}^{N}$ is a countable finite family of real sequences in $[0,1]$ satisfying the following:

(i) $\varsigma_{n, 1}>\lambda>\max \left\{\lambda_{i}\right\}_{i=1}^{N} ; \varsigma_{n, i}<\varsigma<1$, for each $i$.

(ii) $\lim \inf _{n \longrightarrow \infty} \varsigma_{n, i} \prod_{j=1}^{i-1}\left(1-\varsigma_{n, j}\right)\left(\varsigma_{n, 1}-\lambda_{i-1}\right)>0$, $i=2,3, \ldots, N$.

(iii) $\liminf \operatorname{in}_{n \longrightarrow \infty} \prod_{j=1}^{N}\left(1-\varsigma_{n, j}\right)\left(\varsigma_{n, 1}-\lambda_{N}\right)>0$.

Also, if, in addition, $S_{i}$ is L-Lipschitzian and satisfies condition (1) for each $i$, then $\left\{x_{n}\right\}$ converges strongly to $q \in \cap{ }_{i=1}^{N} F\left(S_{i}\right)$.

Proof. Setting $x_{n+1}=y, \quad x_{n}=t, \quad p=u, \quad k=1$, and $y_{n, N} \in S_{N} x_{n}=v$ in Proposition 3, we obtain

$$
\begin{aligned}
&\left\|x_{n+1}-p\right\|^{2} \leq \varsigma_{n, 1} \\
&\left\|x_{n}-p\right\|^{2}+\sum_{i=2}^{N} \varsigma_{n, i} \prod_{j=1}^{i-1}\left(1-\varsigma_{n, j}\right)\left\|y_{n,(i-1)}-p\right\|^{2} \\
&+\prod_{j=1}^{N}\left(1-\varsigma_{n, j}\right)\left\|y_{n, N}-p\right\|^{2} \\
&-\varsigma_{n, 1}\left[\sum_{i=2}^{N} \varsigma_{n, i} \prod_{j=1}^{i-1}\left(1-\varsigma_{n, j}\right)\left\|x_{n}-y_{n, i-1}\right\|^{2}\right. \\
&\left.+\prod_{j=1}^{N}\left(1-\varsigma_{n, j}\right)\left\|x_{n}-y_{n, N}\right\|^{2}\right] .
\end{aligned}
$$

Applying type-one demicontractive condition on each $S_{i}$, we obtain

$$
\begin{aligned}
\left\|x_{n+1}-p\right\|^{2} \leq & \varsigma_{n, 1}\left\|x_{n}-p\right\|^{2}+\sum_{i=2}^{N} \varsigma_{n, i} \prod_{j=1}^{i-1}\left(1-\varsigma_{n, j}\right)\left[\left\|x_{n}-p\right\|^{2}+\lambda_{i-1}\left\|x_{n}-y_{n,(i-1)}\right\|^{2}\right] \\
& +\prod_{j=1}^{N}\left(1-\varsigma_{n, j}\right)\left[\left\|x_{n}-p\right\|^{2}+\lambda_{N}\left\|x_{n}-y_{n, N}\right\|^{2}\right] \\
& -\varsigma_{n, 1}\left[\sum_{i=2}^{N} \varsigma_{n, i} \prod_{j=1}^{i-1}\left(1-\varsigma_{n, j}\right)\left\|x_{n}-y_{n, i-1}\right\|^{2}+\prod_{j=1}^{N}\left(1-\varsigma_{n, j}\right)\left\|x_{n}-y_{n, N}\right\|^{2}\right] \\
= & {\left[\varsigma_{n, 1}+\sum_{i=2}^{N} \varsigma_{n, i} \prod_{j=1}^{i-1}\left(1-\varsigma_{n, j}\right)+\prod_{j=1}^{N}\left(1-\varsigma_{n, j}\right)\right]\left\|x_{n}-p\right\|^{2} } \\
& -\left(\varsigma_{n, 1}-\lambda_{i-1}\right) \sum_{i=2}^{N} \varsigma_{n, i} \prod_{j=1}^{i-1}\left(1-\varsigma_{n, j}\right)\left\|x_{n}-y_{n, i-1}\right\|^{2} \\
& -\left(\varsigma_{n, 1}-\lambda_{N}\right) \prod_{j=1}^{N}\left(1-\varsigma_{n, j}\right)\left\|x_{n}-y_{n, N}\right\|^{2} .
\end{aligned}
$$


Consequently, if we set $k=1$ in Proposition 2, we obtain

$$
\begin{aligned}
\left\|x_{n+1}-p\right\|^{2} \leq & \left\|x_{n}-p\right\|^{2}-\left[\sum_{i=2}^{N} \varsigma_{n, i} \prod_{j=1}^{i-1}\left(1-\varsigma_{n, j}\right)\left(\varsigma_{n, 1}-\lambda_{i-1}\right)\right. \\
& \cdot\left\|x_{n}-y_{n, i-1}\right\|^{2} \\
& \left.+\prod_{j=1}^{N}\left(1-\varsigma_{n, j}\right)\left(\varsigma_{n, 1}-\lambda_{N}\right)\left\|x_{n}-y_{n, N}\right\|^{2}\right] .
\end{aligned}
$$

Furthermore, condition (i) on the control sequences implies that $\lim _{n \longrightarrow \infty}\left\|x_{n}-p\right\|$ exists; hence, $\left\{x_{n}\right\}$ is bounded. Similarly, conditions (ii) and (iii) imply that $\lim _{n \longrightarrow \infty}\left\|x_{n}-y_{n, i}\right\| 0, i=1,2, \ldots, N$. Finally, the demiclosedness property of each $\left(I-S_{i}\right)$, boundedness of $\left\{x_{n}\right\}$, uniqueness of the limit of a weakly convergent sequence, and Opial property of a real Hilbert space guarantee the weak convergence of $\left\{x_{n}\right\}$ to $q \in \cap_{i=1}^{N} F\left(S_{i}\right)$. Also, since $S_{i}$ is LLipschitzian and satisfies condition (1) for each $i$, it then follows from standard argument that $\left\{x_{n}\right\}$ converges strongly to $q \in \cap_{i=1}^{N} F\left(S_{i}\right)$.

Remark 2. If $N=2$ and we set $\varsigma_{n, 1}=\varsigma_{n}$ and $\varsigma_{n, 2}=\beta$, for all $n$, $I$ (the ide ntity mapping) $=S_{1}$, and $S=S_{2}$, we obtain

$$
\begin{aligned}
x_{n+1} & =\varsigma_{n, 1} x_{n}+\sum_{i=2}^{2} \varsigma_{n, i} \prod_{j=1}^{i-1}\left(1-\varsigma_{n, j}\right) S_{n, i-1}+\prod_{j=1}^{2}\left(1-\varsigma_{n, j}\right) S_{n, 2} \\
& =\varsigma_{n} x_{n}+\left(1-\varsigma_{n}\right) \beta_{n} I x_{n}+\left(1-\varsigma_{n}\right)\left(1-\beta_{n}\right) S x_{n} \\
& =\varsigma_{n} x_{n}+\left(1-\varsigma_{n}\right)\left[\beta x_{n}+(1-\beta) S x_{n}\right],
\end{aligned}
$$

which was considered by Osilike and Isiogugu [10].

\section{Applications}

We now present the application of Theorem 5 in the construction of algorithms for approximating a common solution of an equilibrium problem and fixed point problem.

For solving the equilibrium problems for a bifunction $F: C \times C \longrightarrow \mathbb{R}$, let us assume that $F$ satisfies the following conditions:

(A1): $F(g, g)=0$ for all $g \in C$

(A2): $F$ is monotone, that is, $F(g, h)+F(h, g) \leq 0$, for all $g, h \in C$

(A3): for each $g, h, z \in C, \lim _{t \downarrow 0} F(t z+(1-t) g, h) \leq$ $F(g, h)$

(A4): for each $g \in C, h \mapsto F(g, h)$ is convex and lower semicontinuous
Lemma 2 (see [31]). Let $C$ be a nonempty convex closed subset of a real Hilbert space $H$ and $F: C \times C \longrightarrow \mathbb{R}$, a bifunction satisfying (A1)-(A4). Let $r>0$ and $g \in H$. Then, there exists $z \in C$ such that

$$
F(z, h)+\frac{1}{r}\langle h-z, z-g\rangle \geq 0, \quad \forall h \in C .
$$

Lemma 3 (see [32]). Let $C$ be a nonempty convex closed subset of a real Hilbert space $H$. Assume that $F: C \times C \longrightarrow \mathbb{R}$ satisfies (A1)-(A4). Let $r>0$ and $g \in H$. Define $T_{r}: H \longrightarrow 2^{C}$ by

$$
T_{r}(g)=\left\{z \in C: F(z, h)+\frac{1}{r}\langle h-z, z-g\rangle \geq 0\right\}, \quad \forall h \in C .
$$

Then, the following hold:

(1) $T_{r}$ is single valued.

(2) $T_{r}$ is firmly nonexpansive, that is, for any $g, h \in H$, $\left\|T_{r} g-T_{r} h\right\|^{2} \leq\left\langle T_{r} g-T_{r} h, g-h\right\rangle$.

(3) $F\left(T_{r}\right)=E P(F)$.

(4) $E P(F)$ is convex and closed.

Lemma 4 (see [33]). Let $C$ be a nonempty convex closed subset of a real Hilbert space $H$ and $F: C \times C \longrightarrow \mathbb{R}$, a bifunction satisfying (A1)-(A4). Let $r>0$ and $g \in H$. Then, for all $g \in H$ and $p \in F\left(T_{r}\right)$,

$$
\left\|p-T_{r} g\right\|^{2}+\left\|T_{r} g-g\right\|^{2} \leq\|p-g\|^{2} .
$$

Lemma 5. Let $H$ be a real Hilbert space, and let $C$ be a nonempty convex closed subset of $H$. Let $P_{C}$ be the convex projection onto $C$. Then, convex projection is characterized by the following relations:

(i) $g^{*}=P_{C}(g) \Leftrightarrow\left\langle g-g^{*}, h-g^{*}\right\rangle \leq 0$, for all $h \in C$.

(ii) $\left\|g-P_{C} g\right\|^{2} \leq\|g-h\|^{2}-\left\|h-P_{C} g\right\|^{2}$.

(iii) $\left\|g-P_{C} h\right\|^{2} \leq\|g-h\|^{2}-\left\|P_{C} h-h\right\|^{2}$.

Motivated by Algorithm 19 of Isiogugu et al. [34], we obtain the following result using a selection of Algorithm 4.2 above in the sense of [34].

Theorem 6. Let $C$ be a nonempty convex closed subset of a real Hilbert space $H, f: C \times C \longrightarrow \mathbb{R}$, a bifunction satisfying (A1)-(A4) and $\left\{T_{i}\right\}_{i=1}^{N}$ be such that $T_{i}: C \longrightarrow P(C)$ is type-one $\lambda_{i}$-strictly pseudo-contractive-type mappings, and $\left(I-T_{i}\right)$ is weakly demiclosed at zero for each $i=1,2, \ldots, N$. Suppose that $\mathbb{F}=\cap_{i=1}^{N} F_{s}\left(T_{i}\right) \cap E P(f) \neq \varnothing$. Let $\left\{x_{n}\right\}$ be a sequence generated from arbitrary $x_{0} \in C$ as follows: 
Algorithm 1.

$$
\left\{\begin{array}{l}
y_{n}=\varsigma_{n, 1} x_{n}+\sum_{i=2}^{N} \varsigma_{n, i} \prod_{j=1}^{i-1}\left(1-\varsigma_{n, j}\right) y_{n, i-1}+\prod_{j=1}^{N}\left(1-\varsigma_{n, j}\right) y_{n, N}, \\
u_{n} \in K \text { such that } F\left(u_{n}, y\right)+\frac{1}{r_{n}}\left\langle y-u_{n}, u_{n}-y_{n}\right\rangle \geq 0, \quad \forall y \in K, \\
x_{n+1}=\frac{1}{2}\left(u_{n}+x_{n}\right),
\end{array}\right.
$$

where $y_{n, i} \in T_{i} x_{n}$ for each $i$ and $\left\{\left\{\varsigma_{n, i}\right\}_{n=1}^{\infty}\right\}_{i=1}^{N}$ is a finite family of real sequences in $[0,1]$ for each $i$ satisfying (i) $\varsigma_{n, 1}>\lambda>\max \left\{\lambda_{i}\right\}_{i=1}^{N}$; $\varsigma_{n, i}<\varsigma<1$, for each $i$.

(ii) $\liminf \operatorname{in}_{n \longrightarrow \infty} \varsigma_{n, i} \prod_{j=1}^{i-1}\left(1-\varsigma_{n, j}\right)\left(\varsigma_{n, 1}-\lambda_{i-1}\right)>0$, $i=2,3, \ldots, N$.

(iii) $\liminf \operatorname{in}_{n \longrightarrow \infty} \prod_{j=1}^{N}\left(1-\varsigma_{n, j}\right)\left(\varsigma_{n, 1}-\lambda_{N}\right)>0$. Also, if, in addition, $T_{i}$ satisfies condition (1) for each $i$,

(iv) $\left\{r_{n}\right\} \subset[a, \infty)$ for some $a>0$.

Then, $\left\{x_{n}\right\}$ converges strongly to $p \in \mathbb{F}$.

\section{Proof}

$$
\begin{aligned}
\left\|x_{n+1}-p\right\|^{2}= & \left\|\frac{1}{2}\left(x_{n}+u_{n}\right)-p\right\|^{2} \\
= & \frac{1}{2}\left\|x_{n}-p\right\|^{2}+\frac{1}{2}\left\|u_{n}-p\right\|^{2}-\frac{1}{4}\left\|x_{n}-u_{n}\right\|^{2} \\
\leq & \frac{1}{2}\left\|x_{n}-p\right\|^{2}+\frac{1}{2}\left\|y_{n}-p\right\|^{2}-\frac{1}{4}\left\|x_{n}-u_{n}\right\|^{2} \\
= & \frac{1}{2}\left\|x_{n}-p\right\|^{2}-\frac{1}{4}\left\|x_{n}-u_{n}\right\|^{2}+\frac{1}{2}\left\|\varsigma_{n, 1} x_{n}+\sum_{i=2}^{N} \varsigma_{n, i} \prod_{j=1}^{i-1}\left(1-\varsigma_{n, j}\right) y_{n, i-1}+\prod_{j=1}^{N}\left(1-\varsigma_{n, j}\right) y_{n, N}-p\right\|^{2} \|^{2} \\
\leq & \frac{1}{2}\left\|x_{n}-p\right\|^{2}-\frac{1}{4}\left\|x_{n}-u_{n}\right\|^{2}+\frac{1}{2}\left[\left\|x_{n}-p\right\|^{2}-\left[\sum_{i=2}^{N} \varsigma_{n, i} \prod_{j=1}^{i-1}\left(1-\varsigma_{n, j}\right)\left(\varsigma_{n, 1}-\lambda_{i-1}\right)\left\|x_{n}-y_{n, i-1}\right\|^{2}\right.\right. \\
& \left.\left.+\prod_{j=1}^{N}\left(1-\varsigma_{n, j}\right)\left(\varsigma_{n, 1}-\lambda_{N}\right)\left\|x_{n}-y_{n, N}\right\|^{2}\right]\right] \\
& \left.+\prod_{j=1}^{N}\left(1-\varsigma_{n, j}\right)\left(\varsigma_{n, 1}-\lambda_{N}\right)\left\|x_{n}-y_{n, N}\right\|^{2}\right] . \\
= & -\frac{1}{4}\left\|x_{n}-u_{n}\right\|^{2}+\left\|x_{n}-p\right\|^{2}-\frac{1}{2}\left[\sum_{i=2}^{N} \varsigma_{n, i} \prod_{j=1}^{i-1}\left(1-\varsigma_{n, j}\right)\left(\varsigma_{n, 1}-\lambda_{i-1}\right)\left\|x_{n}-y_{n, i-1}\right\|^{2}\right. \\
&
\end{aligned}
$$

It then follows that $\lim _{n \longrightarrow \infty}\left\|x_{n}-p\right\|$ exists; hence, $\left\{x_{n}\right\}$ is bounded. Also,

$$
\begin{aligned}
& \sum_{n=1}^{\infty} \frac{1}{2} \varsigma_{n, i} \prod_{j=1}^{i-1}\left(1-\varsigma_{n, j}\right)\left(\varsigma_{n, 1}-\lambda_{i-1}\right)\left\|x_{n}-y_{n, i-1}\right\|^{2} \\
& \quad \leq\left\|x_{0}-p\right\|^{2}<\infty, \quad i=1,2, \ldots, N-1 . \\
& \sum_{n=1}^{\infty} \frac{1}{2} \prod_{j=1}^{N}\left(1-\varsigma_{n, j}\right)\left(\varsigma_{n, 1}-\lambda_{N}\right)\left\|x_{n}-y_{n, N}\right\|^{2} \leq\left\|x_{0}-p\right\|^{2}<\infty .
\end{aligned}
$$

Thus, from (i), (ii), and (iii), we have that $\lim _{n \rightarrow \infty}\left\|x_{n}-y_{n, i}\right\|=0$, for all $i=1,2, \ldots, N$. Furthermore, $\lim _{n \longrightarrow \infty}\left\|x_{n}-u_{n}\right\|=0$. Consequently, $\lim _{n \longrightarrow \infty}\left\|x_{n+1}-x_{n}\right\|^{2}=$ $\lim _{n \rightarrow \infty}\left\|1 / 2\left(x_{n}-u_{n}\right)\right\|^{2}=0$ which implies that $\left\{x_{n}\right\}$ is a Cauchy sequence in $K$. Also, since $K$ is convex and closed, $\left\{x_{n}\right\}$ converges strongly to some $q \in K$. From the Opial condition of $H$ and the demiclosedness property of $T_{i}$, we have that $q \in T_{i} q$, for all $i-1,2, \ldots, N$.

The remaining part of the proof is similar to the method of [34], Theorem 20. Therefore, it is omitted. 
TABLE 1: Sequences of coordinates $\left\{\bar{x}_{t, n}\right\}_{n=1}^{\infty}$ of $\left\{\bar{x}_{n}\right\}_{n=1}^{\infty}=\left\{\left(x_{1, n}, x_{2, n}, \ldots, x_{t, n}, \ldots x_{m, n}\right)\right\}_{n=1}^{\infty}$ for each $t=1,2, \ldots, m$.

\begin{tabular}{|c|c|c|c|c|c|c|c|}
\hline \multicolumn{2}{|c|}{$N=5, x_{t, 1}=10$} & \multicolumn{2}{|c|}{$N=5, x_{t, 1}=-10$} & \multicolumn{2}{|c|}{$N=10, x_{t, 1}=-10$} & \multicolumn{2}{|c|}{$N=10, x_{t, 1}=10$} \\
\hline$n$ & $x_{t, n}$ & $N$ & $x_{t, n}$ & $n$ & $x_{t, n}$ & $n$ & $x_{t, n}$ \\
\hline 1 & -10 & 1 & 10 & 1 & 10 & 1 & -10 \\
\hline 2 & -4.863391744 & 2 & 4.863391744 & 2 & 4.86333914 & 2 & -4.86333914 \\
\hline 3 & -2.426008398 & 3 & 2.426008398 & 3 & 2.42595782 & 3 & -2.42595782 \\
\hline 4 & -1.220221064 & 4 & 1.220221064 & 4 & 1.22018365 & 4 & -1.22018365 \\
\hline 5 & -0.615820431 & 5 & 0.615820431 & 5 & 0.61579556 & 5 & -0.61579556 \\
\hline 6 & -0.311310767 & 6 & 0.311310767 & 6 & 0.31129518 & 6 & -0.31129518 \\
\hline 7 & -0.157521707 & 7 & 0.157521707 & 7 & 0.1575123 & 7 & -0.1575123 \\
\hline 8 & -0.079750993 & 8 & 0.079750993 & 8 & 0.07974546 & 8 & -0.07974546 \\
\hline 9 & -0.040392014 & 9 & 0.040392014 & 9 & 0.04038883 & 9 & -0.04038883 \\
\hline 10 & -0.020462938 & 10 & 0.020462938 & 10 & 0.02046113 & 10 & -0.02046113 \\
\hline 11 & -0.01036864 & 11 & 0.01036864 & 11 & 0.01036763 & 11 & -0.01036763 \\
\hline 12 & -0.005254554 & 12 & 0.005254554 & 12 & 0.00525399 & 12 & -0.00525399 \\
\hline 13 & -0.002663152 & 13 & 0.002663152 & 13 & 0.00266284 & 13 & -0.00266284 \\
\hline 14 & -0.00134987 & 14 & 0.00134987 & 14 & 0.0013497 & 14 & -0.0013497 \\
\hline 15 & -0.000684252 & 15 & 0.000684252 & 15 & 0.00068416 & 15 & -0.00068416 \\
\hline 16 & -0.000346866 & 16 & 0.000346866 & 16 & 0.00034682 & 16 & -0.00034682 \\
\hline 17 & -0.000175843 & 17 & 0.000175843 & 17 & 0.00017582 & 17 & -0.00017582 \\
\hline 18 & -0.000089144 & 18 & 0.000089144 & 18 & 0.00008913 & 18 & -0.00008913 \\
\hline 19 & -0.000045193 & 19 & 0.000045193 & 19 & 0.00004519 & 19 & -0.00004519 \\
\hline 20 & -0.000022912 & 20 & 0.000022912 & 20 & 0.00002291 & 20 & -0.00002291 \\
\hline 21 & -0.000011615 & 21 & 0.000011615 & 21 & 0.00001161 & 21 & -0.00001161 \\
\hline 22 & -0.000005887 & 22 & 0.000005887 & 22 & 0.00000588 & 22 & -0.00000588 \\
\hline 23 & -0.000002983 & 23 & 0.000002983 & 23 & 0.00000298 & 23 & -0.00000298 \\
\hline 24 & -0.000001509 & 24 & 0.000001509 & 24 & 0.00000151 & 24 & -0.00000151 \\
\hline 25 & -0.000000762 & 25 & 0.000000762 & 25 & 0.00000076 & 25 & -0.00000076 \\
\hline 26 & -0.000000384 & 26 & 0.000000384 & 26 & 0.00000038 & 26 & -0.00000038 \\
\hline 27 & -0.000000191 & 27 & 0.000000191 & 27 & 0.00000019 & 27 & -0.00000019 \\
\hline 28 & -0.000000095 & 28 & 0.000000095 & 28 & 0.00000009 & 28 & -0.00000009 \\
\hline 29 & -0.000000046 & 29 & 0.000000046 & 29 & 0.00000004 & 29 & -0.00000004 \\
\hline 30 & -0.00000002 & 30 & 0.00000002 & 30 & 0.00000002 & 30 & -0.00000002 \\
\hline 31 & -0.000000007 & 31 & 0.000000007 & 31 & 0.00000001 & 31 & -0.00000001 \\
\hline 32 & 0 & 32 & 0 & & & & \\
\hline
\end{tabular}

\section{Examples}

We present the numerical computation of the iteration scheme of Theorem 5 .

Let $H=\left(\mathbb{R}^{m},\|\|,. \leq\right) R^{m}$ with the usual norm "\|.\|" on $R^{m}$ and partial order " $\leq$ " on $\mathbb{R}, C=\left\{\bar{x}=\left(x_{1}, x_{2}, \ldots\right.\right.$, $\left.\left.x_{t}, \ldots, x_{m}\right) \in \mathbb{R}^{m}: x_{1}=x_{2}=\ldots=x_{t}=\ldots=x_{m}\right\}$. Observe that $(C,\|\|,. \leq)$ is a convex closed linear total ordered subset of $R^{n}$ with $\bar{a} \leq \bar{b}$ if and only if $a_{t} \leq b_{t}$ for all $t=1,2,3, \ldots, m$. Denote the order interval $\bar{a} \leq \bar{x} \leq \bar{b}$ by $[\bar{a}, \bar{b}]$, and let $\left\{S_{i}\right\}_{i=1}^{\infty}$ be a countable infinite family of mappings and $S_{i}: C \longrightarrow C B(C)$ define for each $i$ and $\bar{x} \in C$ by

$$
S_{i} \bar{x}= \begin{cases}{\left[-\left(\frac{4 i}{2 i+1}\right) \bar{x},-\left(\frac{3 i}{2 i+1}\right) \bar{x}\right],} & \bar{x} \geq \overline{0}, \\ {\left[-\left(\frac{3 i}{2 i+1}\right) \bar{x},-\left(\frac{4 i}{2 i+1}\right) \bar{x}\right],} & \bar{x}<\overline{0} .\end{cases}
$$

Clearly, for each $i$,

$$
\begin{aligned}
& \text { (I) } F\left(S_{i}\right)=\{\overline{0}\} . \\
& \text { (II) } P_{S_{i}} \bar{x}=\{-(3 i /(2 i+1)) \bar{x}\} .
\end{aligned}
$$

$$
\mathscr{D}\left(S_{i} \bar{x}, S_{i} \bar{y}\right)= \begin{cases}\frac{4 i}{2 i+1}\|\bar{x}-\bar{y}\|, & \bar{x}, \bar{y} \geq \overline{0}, \\ \frac{4 i}{2 i+1}\|\bar{x}-\bar{y}\|, & \bar{x}, \bar{y}<\overline{0}, \\ \left\|\frac{3 i}{2 i+1} \bar{x}-\frac{4 i}{2 i+1} \bar{y}\right\| \geq\left\|\frac{3 i}{2 i+1} \bar{x}-\frac{3 i}{2 i+1} \bar{y}\right\|, & \bar{x} \geq \overline{0}, \bar{y}<\overline{0} .\end{cases}
$$




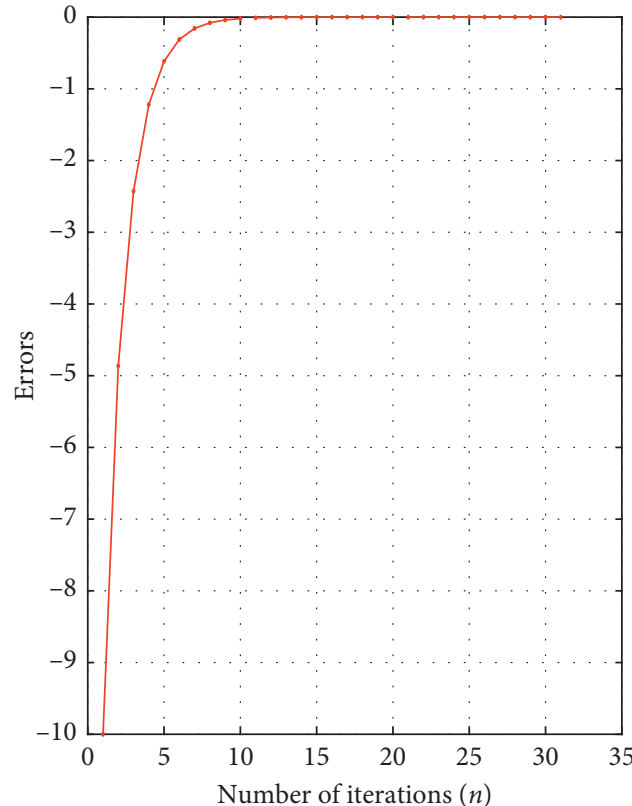

$\longrightarrow$ Case 1a: $N=5$ and $x_{t, 1}=10$

(a)

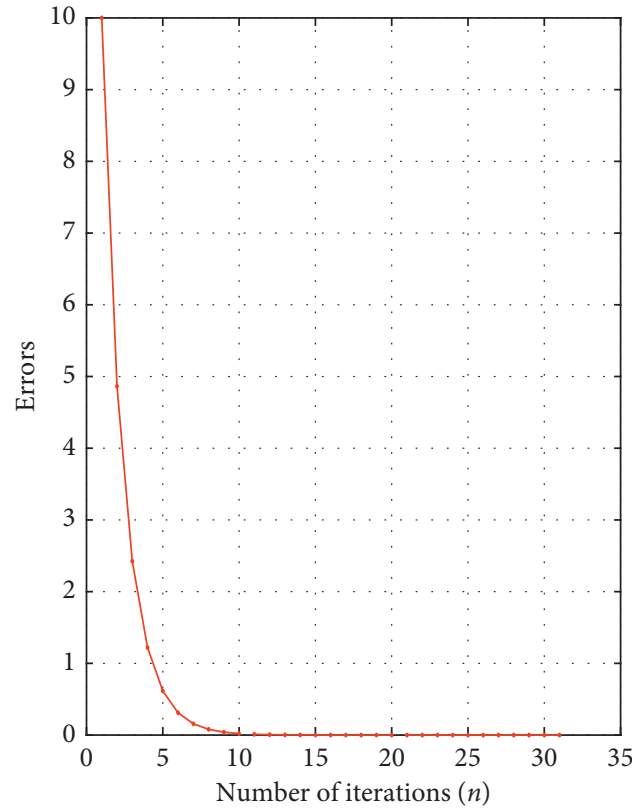

- Case 2a: $N=10$ and $x_{t, 1}=-10$

(c)

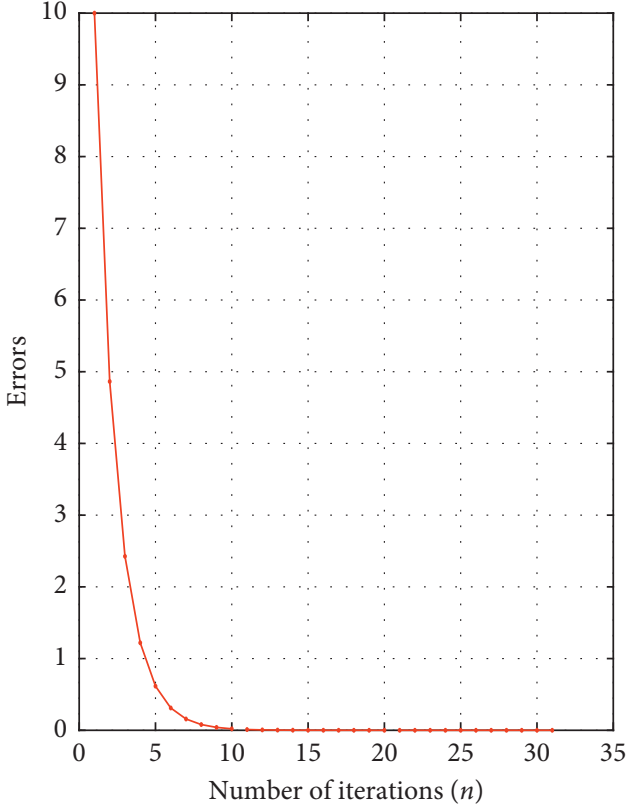

- Case 1b: $N=5$ and $x_{t, 1}=-10$

(b)

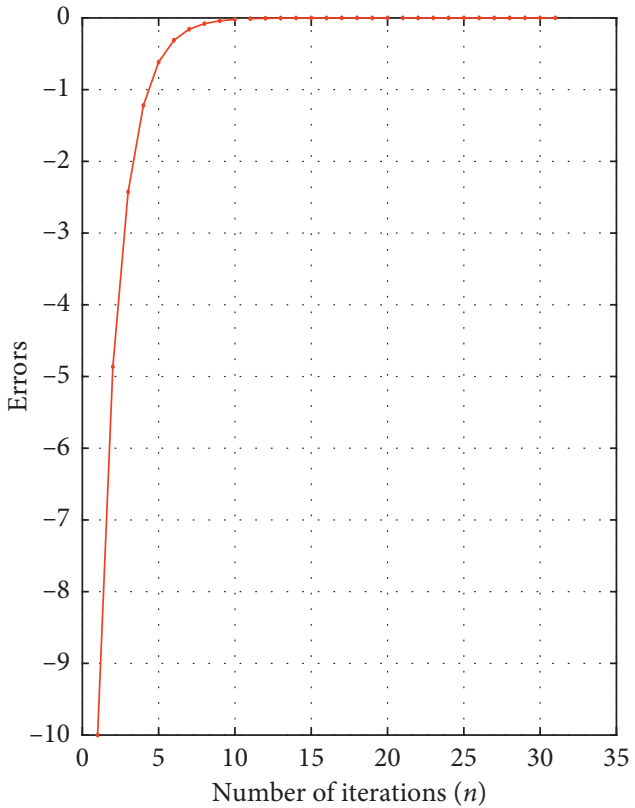

- Case 2b: $N=10$ and $x_{t, 1}=10$

(d)

Figure 1: Errors vs. iteration numbers $(n)$ : case 1a (a); case $1 \mathrm{~b}(\mathrm{~b})$; case $2 \mathrm{a}(\mathrm{c})$; case $2 \mathrm{~b}(\mathrm{~d})$.

(III) $\|\bar{u}-\bar{v}\|=(3 i /(2 i+1))\|\bar{x}-\bar{y}\| \leq \mathscr{D}\left(S_{i} \bar{x}, S_{i} \bar{y}\right)$, for all $\bar{u} \in P_{S_{i}} \bar{x}, \bar{v} \in P_{S_{i}} \bar{y}$.

(IV) $\cap \cap_{i=1}^{N} F\left(S_{i}\right)=\{\overline{0}\}$.

(V) $\quad \mathrm{d}^{2}\left(\bar{x}, S_{i} \bar{x}\right)=\|\bar{x}-(-(3 i /(2 i+1)) \bar{x})\|^{2}=\| \bar{x}+(3 i /(2 i+$ 1)) $\bar{x}\left\|^{2}=((5 i+1) /(2 i+1))^{2}\right\| \bar{x} \|^{2}$.

(VI) $d\left(\bar{x}, F\left(S_{i}\right)\right)=d(\bar{x},\{\overline{0}\})=\|\bar{x}\|$.
(VII) $H^{2}\left(S_{i} \bar{x}, S \overline{0}\right)=\|((4 i / 2 i+1)) \bar{x}\|^{2}=\|\bar{x}\|^{2}+[((4 i / 2 i+$ 1) $\left.)^{2}-1\right]\|\bar{x}\|^{2}=\|\bar{x}\|^{2}+\left(12(i)^{2}-4 i-1 /(2 i+1)^{2}\right)\|\bar{x}\|^{2}$.

It then follows from $(\mathrm{V})$ and (VII) that

(VIII) $H^{2}\left(S_{i} \bar{x}, S \overline{0}\right)=\|\bar{x}-\overline{0}\|^{2}+\left(12(i)^{2}-4 i-1 / 25(i)^{2}\right.$ $+10 i+1) \mathrm{d}^{2}\left(\bar{x}, S_{i} \bar{x}\right)$.

Also, from (V) and (VI), we obtain that 
(IX) $\quad d\left(\bar{x}, S_{i} \bar{x}\right) \geq f\left(d\left(\bar{x}, F\left(S_{i}\right)\right)\right)$, where $f:[0, \infty)$ $\longrightarrow[0, \infty)$ is defined by $f(r)=r$.

In summary, for each $i$, we have from (III), (VIII), and (IX) that $S_{i}$ is type-one demicontractive mapping with contraction coefficient $\lambda_{i}=\left(12(i)^{2}-4 i-1 / 25(i)^{2}+10 i+1\right)$ and satisfies condition (1).

Observe that $\sup \left\{\lambda_{i}\right\}_{i=1}^{\infty}=(12 / 25)=\lim _{i \longrightarrow \infty} \lambda_{i}$. Therefore, if we set $-(3 i /(2 i+1)) \bar{x}_{n}=y_{n, i} \in S_{i} \bar{x}_{n}$ and define $\left\{\left\{\varsigma_{n, i}\right\}_{n=1}^{\infty}\right\}_{i=1}^{N} \subseteq 2^{[0,1]}$ by

$$
\varsigma_{n, i}=\frac{38(n i)^{2}+37}{50\left[(n i)^{2}+1\right]}
$$

then

$$
\text { (i) } \begin{aligned}
\varsigma_{n, 1}>(37 / 50) & >(12 / 25)=\sup \left\{\lambda_{i}\right\}_{i=1}^{\infty} \text {; } \\
& \varsigma_{n, i}<(38 / 50)<1 .
\end{aligned}
$$

(ii) $\lim \inf _{n \longrightarrow \infty} \varsigma_{n, i} \prod_{j=1}^{i-1}\left(1-\varsigma_{n, j}\right)\left(\varsigma_{n, 1}-\lambda_{i-1}\right)=$ $\lim _{n \longrightarrow \infty} \varsigma_{n, i} \prod_{j=1}^{i-1}\left(1-\varsigma_{n, j}\right)\left(\varsigma_{n, 1}-\lambda_{i-1}\right)=$ $(38 / 50)(1-(38 / 50))^{i-1}\left((38 / 50)-\lambda_{i-1}\right)>0$, $2 \leq i<N-1$.

(iii) $\liminf _{n \rightarrow \infty} \prod_{j=1}^{N}\left(1-\varsigma_{n, j}\right)\left(\varsigma_{n, 1}-\lambda_{i_{N}}\right)=$ $\lim _{n \rightarrow \infty} \prod_{j=1}^{N}\left(1-\varsigma_{n, j}\right)\left(\varsigma_{n, 1}-\lambda_{i_{N}}\right)=$ $(1-(38 / 50))^{N}\left((38 / 50)-\lambda_{i_{N}}\right)>0$.

Table 1 and Figure 1 show the sequences for $N=5$ and $N=10$. The values are rounded up to 9 decimal places.

\section{Conclusion}

A horizontal iteration scheme for the approximation of a common fixed point of a finite family of mappings is introduced in a real Hilbert space. This algorithm does not require the imposition of sum $=1$ on the control sequences. Its applicability in developing other algorithms is demonstrated in Algorithm 1. Furthermore, its computability is also exhibited in our numerical computations presented in Section 5.

\section{Data Availability}

All data generated or analyzed during this study are included in this published article.

\section{Conflicts of Interest}

The authors declare that there are no conflicts of interest.

\section{Acknowledgments}

The first and third authors acknowledge with thanks the bursary and financial support from Department of Science and Technology and National Research Foundation, Republic of South Africa, Center of Excellence in Mathematical and Statistical Sciences (DST-NRF COE-MaSS) (Postdoctoral and Doctoral Bursary) respectively. Opinions expressed and conclusions arrived are those of the authors and are not necessarily to be attributed to the CoE-MaSS. This research was supported by CoE-MaSS BA2018-012.

\section{References}

[1] F. O. Isiogugu, "Demiclosedness principle and approximation theorems for certain classes of multivalued mappings in Hilbert spaces," Fixed Point Theory and Applications, vol. 2013, no. 1, p. 61, 2013.

[2] F. E. Browder and W. V. Petryshyn, "Construction of fixed points of nonlinear mappings in Hilbert space," Journal of Mathematical Analysis and Applications, vol. 20, no. 2, pp. 197-228, 1967.

[3] J. T. Markin, "A fixed point theorem for set valued mappings," Bulletin of the American Mathematical Society, vol. 74, no. 4, pp. 639-641, 1968.

[4] F. O. Isiogugu, P. Pillay, and M. O. Osilike, "On approximation of fixed points of multi-valued quasi-nonexpansive mappings in Hilbert spaces," Journal of Nonlinear and Convex Analysis, vol. 17, no. 7, pp. 1303-1310, 2016.

[5] F. O. Isiogugu and M. O. Osilike, "Convergence theorems for new classes of multivalued hemicontractive-type mappings," Fixed Point Theory and Applications, vol. 2014, no. 1, p. 93, 2014.

[6] T. L. Hicks and J. D. Kubicek, "On the Mann iteration process in a Hilbert space," Journal of Mathematical Analysis and Applications, vol. 59, no. 3, pp. 498-504, 1977.

[7] S. A. Naimpally and K. L. Singh, "Extensions of some fixed point theorems of Rhoades," Journal of Mathematical Analysis and Applications, vol. 96, no. 2, pp. 437-446, 1983.

[8] F. Kohsaka and W. Takahashi, "Fixed point theorems for a class of nonlinear mappings related to maximal monotone operators in Banach spaces," Archiv der Mathematik, vol. 91, no. 2, pp. 166-177, 2008.

[9] F. Kohsaka and W. Takahashi, "Existence and approximation of fixed points of firmly nonexpansive-type mappings in Banach spaces," SIAM Journal on Optimization, vol. 19, no. 2, pp. 824-835, 2008.

[10] M. O. Osilike and F. O. Isiogugu, "Weak and strong convergence theorems for nonspreading-type mappings in Hilbert spaces," Nonlinear Analysis: Theory, Methods \& Applications, vol. 74, no. 5, pp. 1814-1822, 2011.

[11] Y. Shehu and P. Cholamjiak, "Another look at the split common fixed point problem for demicontractive operators," Revista de la Real Academia de Ciencias Exactas, Físicas y Naturales. Serie A. Matemáticas, vol. 110, no. 1, pp. 201-218, 2016.

[12] H. H. Bauschke, "The approximation of fixed points of compositions of nonexpansive mappings in Hilbert space," Journal of Mathematical Analysis and Applications, vol. 202, no. 1, pp. 150-159, 1996.

[13] C. E Chidume, M. E. Okpala, A. U. Bello, and P. Ndambomve, "Convergence theorems for finite family of a general class of multi-valued strictly pseudo-contractive mappings," Fixed Point Theory and Applications, vol. 2015, no. 1, p. 119, 2015.

[14] S. Iemoto and W. Takahashi, "Approximating commom fixed points of nonexpansive mappings and nonspreading mappings in a Hilbert space," Nonlinear Analysis: Theory, Methods \& Applications, vol. 71, pp. 2080-2089, 2009.

[15] H. Zegeye and N. Shahzad, "An algorithm for a common fixed point of a family of pseudocontractive mappings," Fixed Point Theory and Applications, vol. 2013, no. 1, p. 234, 2013.

[16] P. Cholamjiak, A. A. Abdou, and Y. J. Cho, "Proximal point algorithms involving fixed points of nonexpansive mappings in CAT(0) spaces," Fixed Point Theory and Applications, vol. 2015, no. 1, p. 227, 2015. 
[17] S. Suantai, Y. J. Cho, and P. Cholamjiak, “Halpern's iteration for Bregman strongly nonexpansive mappings in reflexive Banach spaces," Computers \& Mathematics with Applications, vol. 64, no. 4, pp. 489-499, 2012.

[18] H. Zegeye and N. Shahzad, “Convergence of Mann's type iteration method for generalized asymptotically nonexpansive mappings," Computers \& Mathematics with Applications, vol. 62, no. 11, pp. 4007-4014, 2011.

[19] H. H. Bauschke and J. M. Borwein, "On projection algorithms for solving convex feasibility problems," SIAM Review, vol. 38, no. 3, pp. 367-426, 1996.

[20] P. L. Combettes, "The foundations of set theoretic estimation," Proceedings of the IEEE, vol. 81, no. 2, pp. 182-208, 1993.

[21] B. Halpern, "Fixed points of nonexpanding maps," Bulletin of the American Mathematical Society, vol. 73, no. 6, pp. 957$962,1967$.

[22] M. O. Osilike and Y. Shehu, "Cyclic algorithm for common fixed points of finite family of strictly pseudocontractive mappings of Browder-Petryshyn type," Nonlinear Analysis: Theory, Methods \& Applications, vol. 70, no. 10, pp. 35753583, 2009.

[23] H. Zegeye and N. Shahzad, "Strong convergence theorems for a common zero of a finite family of -accretive mappings," Nonlinear Analysis: Theory, Methods \& Applications, vol. 66, no. 5, pp. 1161-1169, 2007.

[24] S. Suantai, W. Cholamjiak, and P. Cholamjiak, "An implicit iteration process for solving a fixed point problem of a finite family of multi-valued mappings in Banach spaces," Applied Mathematics Letters, vol. 25, no. 11, pp. 1656-1660, 2012.

[25] Y. Zhang and Y. Guo, "Weak convergence theorems of three iterative methods for strictly pseudocontractive mappings of Browder-Petryshyn type," Fixed Point Theory and Applications, vol. 2008, no. 1, Article ID 672301, 2008.

[26] J. García-Falset, E. Llorens-Fuster, and T. Suzuki, "Fixed point theory for a class of generalized nonexpansive mappings," Journal of Mathematical Analysis and Applications, vol. 375, no. 1, pp. 185-195, 2011.

[27] E. L. Dozo, "Multivalued nonexpansive mappings and opial's condition," Proceedings of the American Mathematical Society, vol. 38, no. 2, pp. 286-292, 1973.

[28] Z. Opial, "Weak convergence of the sequence of successive approximations for nonexpansive mappings," Bulletin of the American Mathematical Society, vol. 73, no. 4, pp. 591-598, 1967.

[29] Y. Song and H. Wang, "Erratum to "mann and ishikawa iterative processes for multivalued mappings in Banach spaces" [computers \& mathematics with applications, 54 (2007), 872-877," Computers \& Mathematics with Applications, vol. 55, no. 12, pp. 2999-3002, 2008.

[30] K. K. Tan and H. K. Xu, "Approximsating fixed points of nonexpansive mappings by the Ishikawa iteration process," Journal of Mathematical Analysis and Applications, vol. 178, no. 2, pp. 301-308, 1993.

[31] E. Blum and W. Oettli, "From optimization and variational inequalities to equilibrium problems," Mathematics Student, vol. 63, pp. 123-145, 1994.

[32] P. L. Combettes and S. A. Hirstoaga, "Equilibrium programming in Hilbert spaces," Journal of Nonlinear and Convex Analysis, vol. 6, pp. 117-136, 2005.

[33] W. Takahashi and K. Zembayashi, "Strong and weak convergence theorems for equilibrium problems and relatively nonexpansive mappings in Banach spaces," Nonlinear
Analysis: Theory, Methods \& Applications, vol. 70, no. 1, pp. 45-57, 2009.

[34] F. O. Isiogugu, P. Pillay, and P. U. Nwokoro, "On computability and applicability of Mann-Reich-Sabach-type algorithms for approximating the solutions of equilibrium problems in Hilbert spaces," Abstract and Applied Analysis, vol. 2018, Article ID 7218487, 9 pages, 2018. 